\title{
Brewing Optimal Coffee
}

\author{
A. Smith and W. T. Lee* \\ School of Computing and Engineering, University of Huddersfield
}

(Dated: November 12, 2020)

\begin{abstract}
Coffee is a beverage enjoyed worldwide and an active area of current research. The brewing of coffee also presents an opportunity to demonstrate the model, simulate and optimise paradigm in action. This is done in the context of brewing cafetière coffee. Using a published model and experimental data we show that an existing model can be parameterised to simulate the results of that experiment. From here an optimisation step can be applied in which the experimental recipe can be changed to improve the quality of the coffee produced in the experiment. This activity is implemented in the NUMBAS platform.
\end{abstract}

\section{INTRODUCTION}

We report an activity we have developed for open days in which students are guided through the steps of the modelling, simulation and optimisation paradigm in the context of brewing coffee. This allows students to see in a very short time how such models are used in real world applications where the aim is not just to understand how a system operates but also to adjust it to make it work better. Using coffee as an example has a number of advantages for this. Firstly it is familiar to students and can safely be consumed by them, so they can experience through the sense of taste how modelling has improved things. Secondly, the fact that as familiar a subject as coffee brewing is still an area of active research allows us to highlight to students just how broad the opportunities available to apply modelling skills are. Finally, there is a published model and data set and a quantitative definition of 'good' coffee.

Legend has it that the stimulating properties of coffee were discovered by a goatherd who noticed that his charges became unusually spirited after consuming the cherries of a certain plant. ${ }^{1}$ This of course was the coffee plant which comes in robusta and arabica versions. Today world coffee consumption stands at over 160 million $60 \mathrm{~kg}$ bags of coffee a year. ${ }^{2}$

In changing from the goats preferred method of consuming coffee to brewing cafetière coffee, one of the ways humans prefer to take this stimulant, a number of additional stages are needed. The cherries are harvested and their seeds, colloquially and below called coffee beans, are extracted. In their natural state coffee beans are a green color, but they are roasted to a dark brown color; this stage introduces some of the more complex flavours into the coffee. The beans are next ground to a small size. A typical size distribution is bimodal with large and fine grain sizes. This has a strong effect on extraction although we will ignore this in our simpler models. Finally the ground coffee is steeped in boiling water, transferring some of the soluble content of the coffee to the water. The resulting solution is the drink known as coffee.

Chemically coffee is incredibly complex, consisting of nearly 2000 chemicals, ${ }^{3}$ a similar level of chemical complexity to wine. These are sometimes grouped into three categories roughly by molecular weight. The lightest groups are volatile aromatics, largely responsible for the smell of coffee. The middle category are complex sugars and alkaloids such as caffeine. The final and heaviest components are large complex sugars responsible for the bitter taste of coffee. This latter group must be present in small amounts to give brewed coffee some character, but in large amounts give it an overwhelmingly bitter taste.

In this paper we focus on brewing of coffee in a cafetiére, however there are numerous other methods of brewing coffee. Instant coffee is made by brewing and then freeze drying the resulting solution, allowing the coffee to be reconstituted by the addition of hot water. In espresso coffee making water is forced through finely ground, densely packed coffee grains at high pressures (6 to 8 atmospheres typically). The most interesting way of making coffee from a physics perspective is probably by the moka pot which exploits similar physics to a geyser to force boiling water through a coffee bed. Many other ways of brewing coffee are used.

A number of attempts have been made to develop mathematical models of the brewing of coffee by various methods. An instrumented moka pot was used to develop a model of the thermodynamics and fluid mechanics of that brewing method, however the physics of extraction were not considered. ${ }^{4,5}$ Partial differential equation models of extraction in espresso were developed by Fasano et al. ${ }^{6}$ These models were set up to consider the multicomponent nature of coffee but as the main aim of the authors was to investigate the properties of the equations themselves, e.g. existence and uniqueness of solutions, they did not attempt to parameterise them. A multiscale model of coffee brewing was developed by Moroney et al. leveraging models of groundwater flow and focussed on filter coffee extraction. ${ }^{7,8}$ An alternative multiscale model based on Li ion batteries has been applied to espresso brewing. ${ }^{9}$ With the exception of the models developed by Fasano et al., standard practice in this area is to treat 'coffee' as a single component substance and describe it in terms of a mass concentration.

The brewing of good coffee is an art rather than a science, however some rough guidelines on how to brew coffee exist in the golden cup formula developed by the Speciality Coffee Association. This guidance refers to 


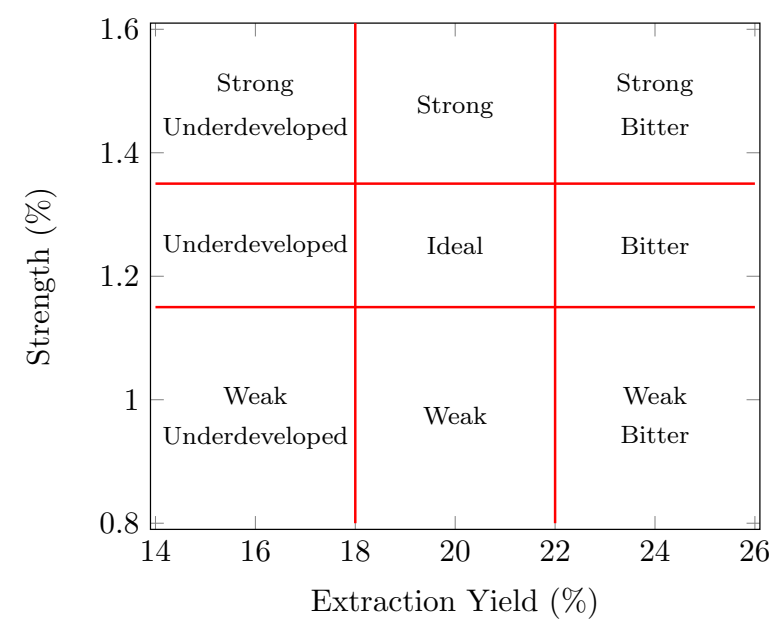

FIG. 1. The coffee brewing control chart, developed by the Speciality Coffee Association. This gives a rough guide to making non-espresso coffee.

brewing methods excluding espresso and is encoded in the coffee brewing control chart. This describes a cup of coffee in terms of two variables: extraction yield and strength. As these form the $x$ - and $y$-axes of the coffee control chart respectively, we denote these by $x$ and $y$. Extraction yield, $x$, is the mass percentage of the coffee grounds that goes into solution. Strength, $y$ is the concentration of the coffee solution expressed as a mass percentage, i.e. the fraction by weight of a cup of coffee that is dissolved coffee solids. Optimal ranges of these quantities were determined through taste tests. ${ }^{10}$ An example coffee control chart appropriate for filter and cafetière coffee is shown in Fig. 1.

The two key assumptions that go into a coffee control chart are that extraction is uniform throughout the coffee bed and the concentration of 'coffee' can be described in terms of a single variable. In fact extraction yield does take into account the multicomponent nature of coffee. A small extraction yield indicates that the chemistry of the dissolved coffee is dominated by low molecular weight volatile compounds, whereas large extraction yields indicate a cup of coffee whose flavour is dominated by high molecular weight bitter tasting chemicals. Some effort has been made to use a coffee control chart to indicate the range of extraction yields occurring, both as a function of grain size and position within the coffee bed. ${ }^{11}$

If the two aforementioned assumptions are accepted then, by conservation of mass, it is easy to see that any coffee brewing process will be described by a straight line. Let $M_{\mathrm{w}}$ and $m$ be, respectively, the mass of water and ground coffee used. If an amount of coffee $\Delta m$ goes into solution then (assuming $\Delta m \ll M_{\mathrm{w}}$ which is true for filter coffee) extraction yield, $x$, is given by $x=\Delta m / m$ and strength, $y$, is given by $y=\Delta m / M_{\mathrm{w}}$. Eliminating $\Delta m$ from the equations for $x$ and $y$ gives $y=\left(m / M_{\mathrm{w}}\right) x$. Thus any brewing process is a straight line passing through the
TABLE I. Coffee concentration data. ${ }^{7}$

Time Concentration

\begin{tabular}{rr}
$(\mathrm{s})$ & $(\mathrm{g} / \mathrm{L})$ \\
\hline 0 & 0.0 \\
36 & 29.1 \\
48 & 31.8 \\
67 & 34.4 \\
149 & 36.3 \\
348 & 36.3 \\
500 & 36.3
\end{tabular}

origin with a gradient equal to the ground coffee to water ratio. The golden cup formula recommends a coffee grounds to water ratio of $30 \mathrm{~g}$ coffee per $\mathrm{kg}$ or litre of water. Of course this in itself does not guarantee good tasting coffee or even that the eventual cup will be in the optimal region of the coffee control chart. Taste will also depend on the brewing time, controlled directly by the operator in the case of a cafetière, but by a range of more complex factors such as grind size distribution in the case of filter coffee or a moka pot.

A published experiment,${ }^{7}$ gave an insight into brewing dynamics under conditions similar to, but not exactly the same as, cafetière brewing. In this experiment coffee grounds and water were mixed at a ratio of $60 \mathrm{~g}$ coffee grounds to 1 litre of water and stirred continuously. This experiment is similar to cafetière brewing but, due to the stirring, the extraction kinetics are likely to be faster. Also the final stage in which the filter is pushed down to trap the grains at the base of the cafetière is not included. At regular intervals during the experiment a small sample of the liquid was extracted and the mass concentration of coffee, $c$, was measured by refractometry (for small concentrations dissolved coffee affects the refractive index of water in a known way). The concentration is the mass of coffee in solution per unit volume of water: $c=\Delta m / V$ where $V=M_{\mathrm{w}} / \rho_{\mathrm{w}}$. (We have assumed that $c$ is small so that the volume of the liquid is not changed by the dissolved coffee.) These data are tabulated in Table I and plotted in Fig. 2.

From the concentration, the extraction yield, $x$, and strength, $y$, can be calculated as shown in Table II. Fig. 3 and Fig. 4 show $x$ and $y$ plotted as functions of time.

We can also plot the $x$ and $y$ data on the coffee control chart. This is shown in Fig. 5. The plot shows that this experiment will produce coffee that is far too strong to be palatable. In the context of the experiment this is reasonable: it is aimed at obtaining scientific data not making good coffee. However, we can ask the question: if the aim was to create good coffee how should the experiment be modified? Specifically: What water to coffee ratio should be used and for how long should the coffee be brewed?

The rest of the paper is organised as follows. In Section II we review the state of the art in modelling the physics and chemistry of coffee extraction, including an 


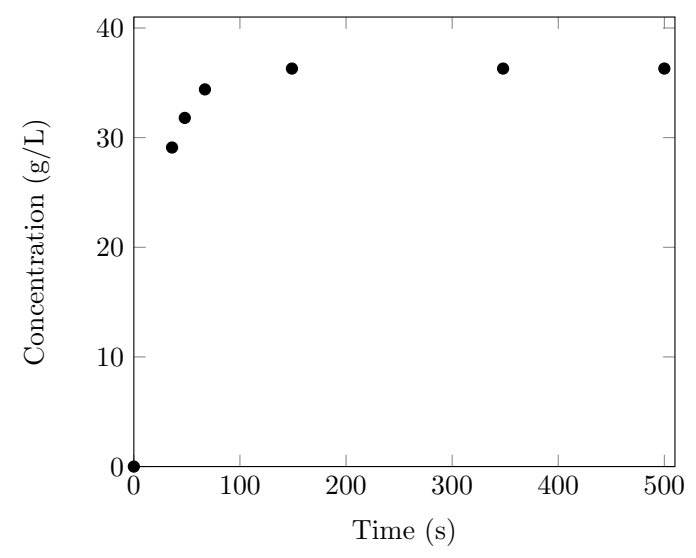

FIG. 2. Brewing data

TABLE II. Extraction yield and strength corresponding to concentrations given in Table I.

\begin{tabular}{rrr} 
Time & Extraction & Strength, \\
$(\mathrm{s})$ & Yield, $x(\%)$ & $y(\%)$ \\
\hline 0 & 0.0 & 0.0 \\
36 & 24.25 & 2.91 \\
48 & 26.50 & 3.18 \\
67 & 28.67 & 3.44 \\
149 & 30.25 & 3.63 \\
348 & 30.25 & 3.63 \\
500 & 30.25 & 3.63
\end{tabular}

application of that modelling framework to the experiment described above, and develop and parameterise a set of equations describing this system. We also investigate what these models tell us about the way to optimise coffee brewing with this experiment. In Section III we discuss how these steps can be simplified to produce an activity students can work through in a relatively short session, this requires simplifying the theory and parame-

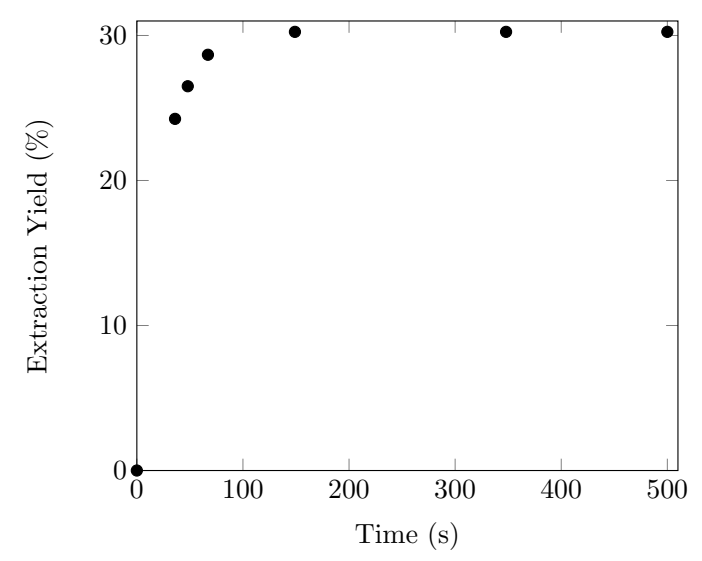

FIG. 3. Plot of extraction yield, $x$ against time.

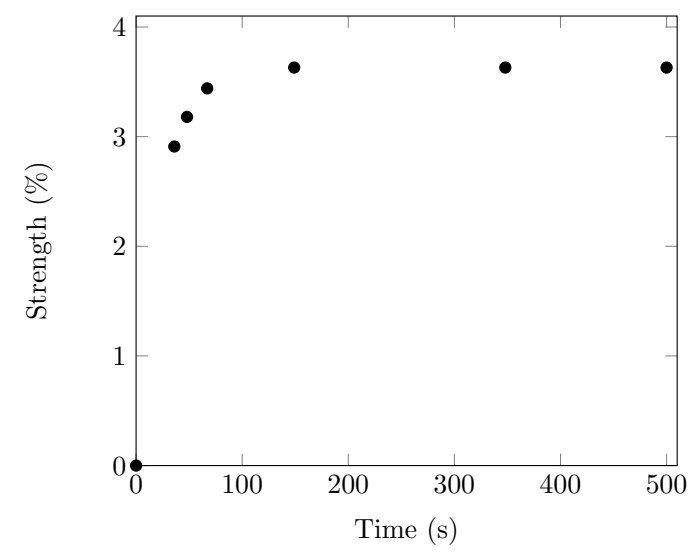

FIG. 4. Plot of coffee strength, $y$ against time.

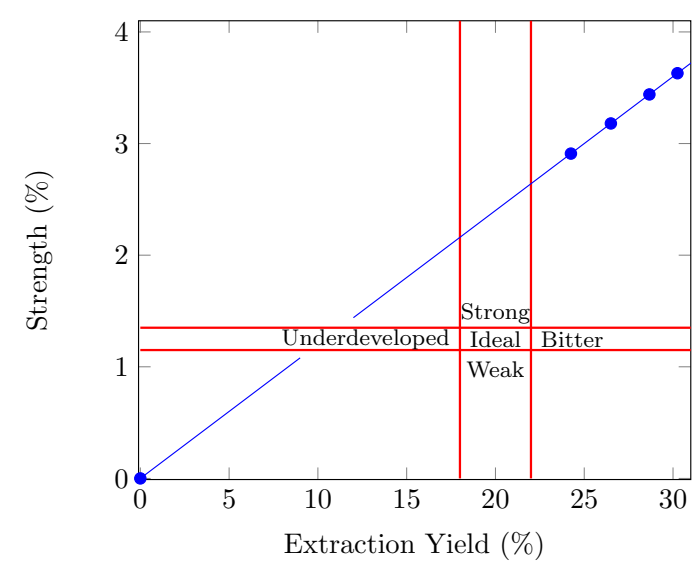

FIG. 5. Experimental data and best fit line plotted on the coffee control chart. This shows that the experiment will brew coffee much too strong to be optimal for human consumption.

terisation of the model considerably. We describe our implementation of an electronic worksheet guiding students through the calculation, and discuss the errors our results have generated. In Section IV we discuss potential extensions of this model. The activity as described is aimed at students entering university, more advanced students with a grasp of curve fitting and differential equations can investigate this system in a lot more depth. Finally, we give conclusions in Section $\mathrm{V}$.

\section{THEORETICAL BACKGROUND}

Building on previous work by Fasano ${ }^{6}$ and models of chemical extraction in groundwater flow, ${ }^{12}$ Moroney et al. developed a set of equations that can describe coffee brewing in both the filter coffee geometry and in the cafetière experiment described above. These equations track coffee in solid and dissolved forms within large and small coffee grains and in dissolved form in pores between 
grains. Moroney et al. developed these equations for filter coffee initially and then, by neglecting convection and diffusion terms, applied them to the cafetière experiment.

These equations can be reformulated as a compartment model tracking masses of soluble coffee present in large grains, $m_{1}$, small grains, $m_{2}$, and dissolved in the water $m_{\mathrm{w}}$.

$$
\begin{aligned}
\dot{m}_{1} & =-\frac{m_{1}}{\tau_{1}} \\
\dot{m}_{2} & =-\frac{m_{2}}{\tau_{2}} \\
\dot{m}_{\mathrm{w}} & =-\dot{m}_{1}-\dot{m}_{2} \\
m_{1} & =m_{10} \text { at } t=0 \\
m_{2} & =m_{20} \text { at } t=0 \\
m_{\mathrm{w}} & =0 \text { at } t=0
\end{aligned}
$$

One simplifying assumption we have made is to assume that the coffee is very weak so the coffee already in solution does not hinder extraction. These equations have solutions

$$
\begin{aligned}
& m_{1}=m_{10} e^{-t / \tau_{1}} \\
& m_{2}=m_{20} e^{-t / \tau_{2}} \\
& m_{\mathrm{w}}=m_{10}\left(1-e^{-t / \tau_{1}}\right)+m_{20}\left(1-e^{-t / \tau_{2}}\right)
\end{aligned}
$$

If $m$ is the total (initial) mass of ground coffee and $M_{\mathrm{w}}$ is the initial mass of water, corresponding to a volume of water $V_{\mathrm{w}}=M_{\mathrm{w}} / \rho_{\mathrm{w}}$ then

$$
\begin{aligned}
x & =\frac{m_{\mathrm{w}}}{m} \\
y & =\frac{m_{\mathrm{w}}}{M_{\mathrm{w}}} \\
c & =\frac{m_{w}}{V_{\mathrm{w}}}
\end{aligned}
$$

where $x$ is extraction yield, $y$ is strength and $c$ is concentration. Note that $m \neq m_{10}+m_{20}$ because not all the mass of the grains is soluble whereas $m_{10}$ and $m_{20}$ are soluble masses.

\section{ACTIVITY}

An open day activity aimed at visiting school students considering attending our university was developed based on this data. In this activity we ask students how the experiment described above should be modified to produce drinkable coffee. This works out as asking two questions: what is the coffee to water ratio that should be used and how long should the brewing have been carried out for? To address these questions at an accessible level and in a relatively short amount of time we simplify the theory considerably. In the next section we discuss possible extensions to the theory that could be of interest to educators wishing to adapt the activity at a more advanced level or providing extension questions to more able students.

To make it possible to let students complete the activity within the relatively short time available several simplifications are used

- Coffee extraction is considered to be a single timestep process so that the extraction equations become $x=x_{0}(1-\exp (-t / \tau))$, and $y$ having a similar structure. This is equivalent to considering that coffee grains are monodisperse rather than separated into small and large grains.

- Students are not expected to solve differential equations, solutions are provided. Furthermore, as noted above, we neglect the effect of hindering of coffee extraction by coffee already in solution, i.e. we will assume that when we change the water to coffee ratio the equation for $x$ and its parameters will not change.

The students are guided through the following steps. Firstly they are introduced to the coffee control chart and asked to interpret some data. They are given $x$ and $y$ coordinates and asked to use the coffee control chart to interpret these coordinates in terms of the taste of coffee. Then they are given some data for initial and final mass of coffee grains and mass of water used and asked to calculate $x$ and $y$ and interpret these as the taste of the resulting coffee.

In the next step students are introduced to the French press data and the aim of the activity. The French press data is presented in its original form as concentration data. Students are asked to convert some randomly chosen points into $x$ and $y$ coordinates. Following this students are given a complete data set and the equations for $x$ and $y$ (simplified to a single timescale as discussed above). Students are then asked to estimate the parameters $x_{0}$ and $\tau$ by using the final timepoint as an estimate of $x_{0}$ and the first nonzero time point to find $\tau$. These result in the estimates $x_{0}=30.25 \%$ and $\tau=22.25 \mathrm{~s}$. The resulting fit to the data is shown in Fig. 6 .

Using this parameterisation, students then solve the equation for $x$ at the optimal extraction level of $20 \%$ for time, $t=24.08 \mathrm{~s}$. They are then asked to calculate the corresponding value of $y(2.4 \%)$. The next step is to consider what these coordinates mean in terms of the taste of coffee with reference to the coffee brewing control chart. Students should correctly deduce that this coffee is far too strong: so much so that it will not even appear on the chart as it is usually plotted.

Having completed the simulation step students are now asked to move onto the optimisation step. Making the assumption that the parameterisation chosen for the $x(t)$ equation will not change if the water to coffee ratio changes, students are asked to calculate the water to coffee ratio that would result in optimal coffee. (This should come out to be about $31.25 \mathrm{~g}$.) The effect of changing the mass of coffee on the strength is shown in Fig. 7. 


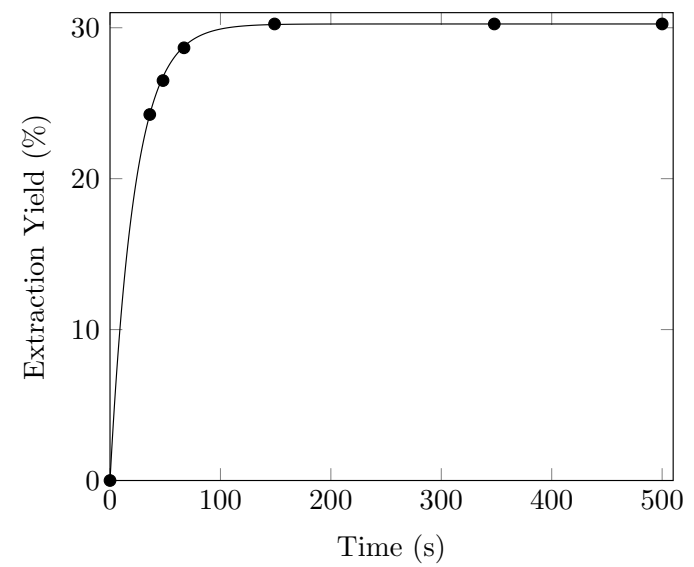

FIG. 6. Algebraic fit to extraction yield, $x$, data. The first and last points are used to estimate the timescale $\tau$ and saturation point $x_{0}$.

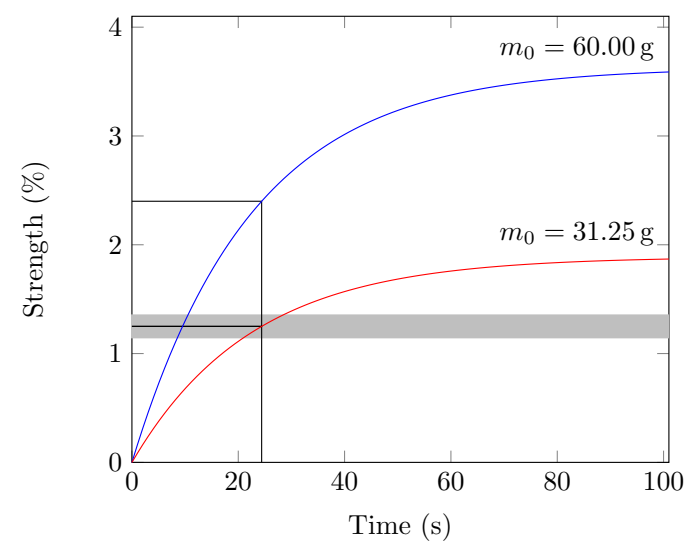

FIG. 7. Adjusting the coffee to water ratio to improve taste. Strength is shown plotted against time with the time at which the extraction yield is optimal shown by a vertical line. The optimal strength is shown in grey. The blue line shows strength as a function of time for the original mass of coffee. The red line shows that if the mass of coffee used is changed to $31.25 \mathrm{~g}$ optimal coffee can be brewed.

With the mathematical side of things complete we now move to an experimental test. Using some scales, a kettle, some ground coffee and two cafetières, two different coffees are brewed. (A student can be asked to confirm the masses are correct or to weigh out the ground coffee, but it is probably safest to let a staff member pour the hot water!) Since cafetières are not continuously stirred, the brewing time students have calculated will not be accurate and it is better to wait the recommended three minutes for the coffee to brew. During this period of brewing you can go over some of the ideas for extensions discussed below in section IV. A volunteer is then sought to taste the too strong coffee and the 'optimal' coffee. The volunteer (on open days usually a parent) typically agrees that the optimal coffee is best.

Several simplifying assumptions were introduced which affect the results to various extents. From the data it seems reasonable that the last data point does indeed correspond to the saturation level so this is not expected to introduce any errors. The assumption that the timescale $\tau$ can be calculated from the first data point is only as accurate as the assumption that the single exponential process accurately describes the experimental data: as we have seen above this is not true. More accurate fitting procedures are discussed in section IV. Note however that this will not affect the optimal ratio of coffee to water as this is independent of the exact form of the extraction curve used.

This activity was implemented within the NUMBAS platform. ${ }^{13,14} \mathrm{~A}$ version of the activity that can be run on a (networked) webbrowser is available from this link. Editable versions of the questions can be accessed on the NUMBAS website and are released under a creative commons licence allowing any modifications. The NUMBAS platform is an interactive environment for implementing mathematical questions and accepts algebraic as well as numerical answers to questions. It has the ability to randomise numerical parameters within a question. It also facilitates scaffolding by incorporating undisclosed supporting information which is available on request, thus guiding any struggling students towards a solution. The stages of the activity are as described above.

\section{EXTENSIONS}

This activity is considerably shortened to allow it to be run within an open day session. Numerous extensions are possible. These include a simpler calculation of the coffee to water ratio without the intermediate step of calculating the time, deriving $x(t)$ and $y(t)$ from a differential equation, an improved parameterisation of the model from least squares fitting using the excel solver or another minimisation technique; and visualisation of the data e.g. in an excel chart or by a geogebra widget.

a. A simple ratio derivation. The calculation of the $m / M_{w}$ ratio presented above is slightly too involved if only the optimal brewing ratio is needed and not the brewing time. This can be directly calculated from the relationship between $x$ and $y$ without making any assumptions about the brewing kinetics if they are not of interest. Since coffee solids are conserved we always have $y=m x / M_{\mathrm{w}}$ so by picking optimal values of $x$ and $y$ from the coffee control chart we can instantly determine the correct value of the brewing ratio $m / M_{w}$. If one student finished the material early this is a good bonus question to ask. Alternatively it can be discussed in the interval while the coffee brews.

b. Deriving the Equations In the activity the equations for $x(t)$ and $y(t)$ are produced directly. However these can be derived. The full equations are relatively complex but a simplified model for $x(t)$ as the result of 
a separable differential equation can be derived if the assumption of a single kinetic timescale is maintained. In this case it is assumed that the rate of dissolution, i.e. increase of $x$, is proportional to the soluble content of the coffee grains $x_{0}-x$ where $x_{0}$ is the maximum possible value of $x$ the soluble content of the coffee grains. This leads to $\dot{x} \propto\left(x_{0}-x\right)$ and introducing a timescale, $\tau$, as a constant of proportionality leads to

$$
\begin{aligned}
\frac{d x}{d t} & =\frac{x_{0}-x}{\tau} \\
x & =0 \quad \text { at } t=0
\end{aligned}
$$

which can be integrated to give

$$
\begin{aligned}
& x=x_{0}\left(1-e^{-t / \tau}\right), \\
& y=\frac{m x_{0}}{M_{\mathrm{w}}}\left(1-e^{-t / \tau}\right) .
\end{aligned}
$$

(Where the equation for $y$ is determined by conservation of coffee solids.)

c. Parameterising the equations The parameterisation of the equations described above is relatively quick but not optimal. A better (but more time consuming) approach would be to carry out least squares fitting using, for instance, the excel solver or a statistical environment such as R. This approach, as well as generating a more accurate fit, also allows a more complex function to be used for instance taking into account the bimodal size distribution. Because of the wide spacing of the points the short timescale is difficult to resolve so we adopt a singular perturbation style approach and assume that in an unresolvable boundary layer ${ }^{15}$ the extraction yield jumps rapidly up from zero to some finite value. This results in the equation

$$
x=x_{1}\left(1-e^{-t / \tau}\right)+x_{2}\left(1-e^{t / \epsilon \tau}\right)
$$

where $x_{1}$ is the maximum extraction possible from large grains which occurs over a timescale $\tau$ and $x_{2}$ is the maximum extraction possible from small grains which occurs over a (very small) timescale $\epsilon \tau$. In practice $\epsilon$ cannot be estimated from the data so instead the function $x=x_{1}\left(1-e^{-t / \tau}\right)+x_{2}$ is fitted to all the datapoints except for $t=0$. The resulting parameters as obtained by the LevenbergMarquardt algorithm ${ }^{16}$ are given in Table III and the fit is shown in Fig. 8. This produces a slightly better fit and the remaining calculations (solving for $t_{\text {optimal }}$ and $m / M_{w}$ ) can still be carried out analytically. d. Visualising the data An excel worksheet, or a platform especially designed for mathematical visualisations such as geogebra, can be incorporated into the fitting process. Either could be used to observe the impact of changing the fitted parameters on the quality of the agreement with the data and could also be linked to additional plots demonstrating how the other quantities of interest vary.

TABLE III. Parameters of the mathematical model of coffee brewing obtained by LevenbergMarquardt least squares fitting. The uncertainty in the final digit is given in brackets at the end.

$$
\begin{array}{c|c}
x_{1} & 26.9(2) \\
\tau & 24.1(1) \mathrm{s} \\
x_{2} & 3.3(2) \\
\chi^{2} & 166.3
\end{array}
$$

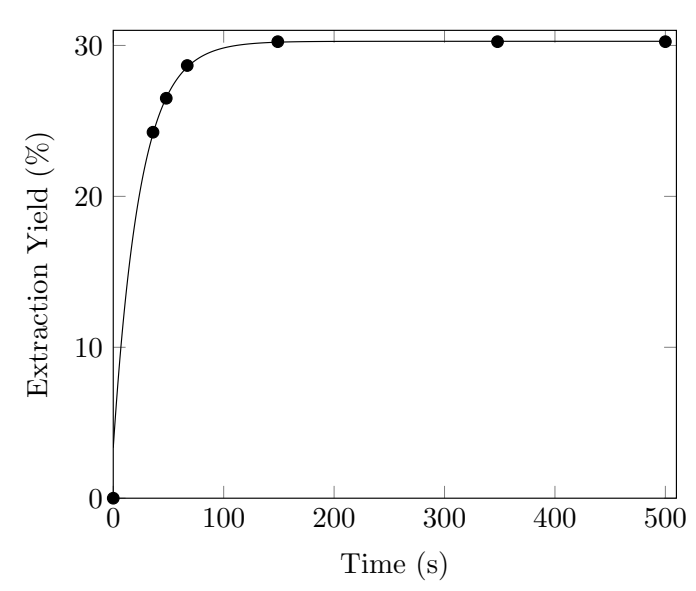

FIG. 8. Least squares fit to data incorporating a boundary layer at the origin.

\section{CONCLUSIONS}

Coffee is an excellent system to introduce students to all the steps of mathematical modelling from setting up a model to using that model to make predictions and improvements and finally to relating findings back to the real world and improving peoples lives one cup of coffee at a time. Coffee is not just an interesting demonstration but also links up to an active area of ongoing research. Implementation of the activity on the NUMBAS platform allows students to work through the calculation at their own pace. *w.lee@hud.ac.uk; Also at MACSI, University of Limerick

${ }^{1}$ K. Sinnott, The Art and Craft of Coffee: An Enthusiast's Guide to Selecting, Roasting, and Brewing Exquisite Coffee (Quarry Books, 2010) iSBN-13: 978-1592535637.
2 International Coffee Association, "World coffee consumption," http://www.ico.org/prices/ new-consumption-table.pdf (2019).

3 M. Petracco, COFFEE Recent Developments; Blackwell Science Ltd.: London, UK (2001). 
${ }^{4}$ C. Gianino, American Journal of Physics 75, 43 (2007).

5 W. D. King, American Journal of Physics 76, 558 (2008).

${ }^{6}$ A. Fasano and F. Talamucci, SIAM Journal on Mathematical Analysis 31, 251 (2000).

7 K. Moroney, W. Lee, S. O'Brien, F. Suijver, and J. Marra, Chemical Engineering Science 137, 216 (2015).

${ }^{8}$ K. M. Moroney, W. T. Lee, S. O. Brien, F. Suijver, and J. Marra, SIAM Journal on Applied Mathematics 76, 2196 (2016).

9 M. I. Cameron, D. Morisco, D. Hofstetter, E. Uman, J. Wilkinson, Z. C. Kennedy, S. A. Fontenot, W. T. Lee, C. H. Hendon, and J. M. Foster, Matter (2020).

10 S. Rao, Everything But Expresso (Independent Publisher, Scott Rao, Available from www.scottrao.com., 2010).

${ }^{11}$ K. M. Moroney, K. OConnell, P. Meikle-Janney, S. B. OBrien, G. M. Walker, and W. T. Lee, PloS one 14 (2019).
12 J. Bear and A. H.-D. Cheng, Modeling groundwater flow and contaminant transport, Vol. 23 (Springer Science \& Business Media, 2010).

13 B. Foster, C. Perfect, and A. Youd, International Journal of e-Assessment 2 (2012).

${ }^{14}$ C. Perfect, in CAA Conference (2015).

15 C. M. Bender and S. A. Orszag, Advanced mathematical methods for scientists and engineers I: Asymptotic methods and perturbation theory (Springer Science \& Business Media, 2013).

16 W. H. Press, S. A. Teukolsky, W. T. Vetterling, and B. P. Flannery, Numerical recipes 3rd edition: The art of scientific computing (Cambridge university press, 2007). 